\title{
1,7-二芳基-1,6-庚二炔化合物的合成
}

\author{
温燕梅* 谢建英冯润光黄绪明 \\ (广东海洋大学理学院 湛江 524088)
}

\begin{abstract}
摘要 1,6 -庚二炔是有机合成的重要中间体, 我们以 $\mathrm{PdCl}_{2} / \mathrm{CuI}$ 为催化剂, $\mathrm{NEt}_{3}$ 为碱, 在四氢呋喃溶剂中实现了二炔与卤 代芳烃 Sonogashira 偶联反应，合成了 10 个 1,7-二芳基-1,6-庚二炔化合物，该方法也适用于二卤芳烃如 1,8-二碘荎与末 端炔偶联合成 1,8 -二苯乙炔基萘. 所有合成的目标化合物结构经 ${ }^{1} \mathrm{H} N \mathrm{NM},{ }^{13} \mathrm{C} N \mathrm{NR}, \mathrm{IR}$ 和 MS 表征.

关键词＼cjkstart钯催化; 1,6-庚二炔; Sonogashira 反应
\end{abstract}

\section{Synthesis of 1,7-Diarylhepta-1,6-diyne Compounds}

\author{
Wen, Yanmei* Xie, Jianying $\quad$ Feng, Runguang $\quad$ Huang, Xuming \\ (College of Science, Guangdong Ocean University, Zhanjiang 524088)
}

\begin{abstract}
Hepta-1,6-diynes are very useful synthetic intermediates. Using $\mathrm{PdCl}_{2} / \mathrm{CuI}$ as the catalyst and $\mathrm{NEt}_{3}$ as the base in tetrahedrofuran, ten 1,7-diarylhepta-1,6-diynes were synthesized by a simple Sonogashira coupling reaction of terminal diacetylenes and aryl halides. It is also indicated that 1,8-diiodonaphthalene can undergo the same transformation to 1,8bis(phenylethynyl)naphthalene under typical conditions. Moreover, the structures of hepta-1,6-diyne derivatives were confirmed by ${ }^{1} \mathrm{H}$ NMR, ${ }^{13} \mathrm{C}$ NMR, IR and MS techniques.
\end{abstract}

Keywords Palladium-catalyzed; Hepta-1,6-diyne; Sonogashira reaction

Sonogashira 反应是过渡金属催化端基炔烃与芳(烯) 基卤化物之间的 $\mathrm{sp}-\mathrm{sp}^{2}$ 碳交叉偶联反应，是形成新的碳 碳键及炔基化的最有效方法之一, 其在取代炔烃以及大 共轭炔烃的合成中得到了广泛的应用 ${ }^{[1]}$. 经过近三十年 的发展，已形成了适用于 Sonogashira 交叉偶联反应的 改良 Pd 催化体系 ${ }^{[2]}$ 和其他的非钯过渡金属催化体系, 诸如 Kanuru 等 ${ }^{[3]}$ 报道的将铑纳米负载在固体载体上, 以 四丁基醋酸胺为碱, 成功地实现了在载体表面催化苯乙 炔与碘苯之间的偶联反应; Wang 等 ${ }^{[4]}$ 报道了以纳米零价 镍和碘化亚铜为共催化剂, 以氢氧化钾为碱, 异丙醇为 溶剂, 加入配体三苯基膦, 在温和的条件下实现了 Sonogashira 偶联反应; 2009 年, Vechorkin 等 ${ }^{[5]}$ 使用二价 镍催化炔烃与不活泼的含 $\beta$-H 烷基卤代物之间的偶联; Liu 等 ${ }^{[6]}$ 报道了铜或铁催化端基炔烃和烯基卤之间的 Sonogashira 偶联.

过渡金属催化不饱和有机化合物合环反应构建环 状结构分子越来越受到人们的关注, 因为过渡金属催化 的反应, 可以在温和的条件下, 合成出结构比较复杂的
化合物. 1,6-二炔化合物是很重要的有机合成中间体, 被广泛地用于过渡金属催化的合环反应合成结构复杂 的多环物质，如五元或六元环的烯酮类化合物 ${ }^{[7]}$ 、二烯 酮类化合物 ${ }^{[8]}$ 、三烯衍生物 ${ }^{[9]}$ 、环外 $1,4-$ 二烯 ${ }^{[10]}$, 具有多 环的功能性分子 1,3-环已二烯衍生物 ${ }^{[11]}$ 、呋喃并五元环 ${ }^{[12]}$ 、苯并呋喃酮衍生物 ${ }^{[13]}$ 、多取代芳胺衍生物 ${ }^{[14]}$ 、稠合 多环二氢香豆素类化合物 ${ }^{[15]}$ 、多元环的苯或具有多元环 的苯的衍生物 ${ }^{[16]}$. 因此发展合成 1,6-二炔的方法和技术 具有重要意义及广阔的应用前景. 本文以 $\mathrm{PdCl}_{2} / \mathrm{CuI}$ 为 催化剂, 三乙胺 $\left(\mathrm{NEt}_{3}\right)$ 为碱, 在四氢呋喃(THF)溶剂中实 现了芳基卤代物与芳基末端二炔 Sonogashira 偶联反应 合成 1,7-二芳基-1,6-庚二炔，该方法也适用于常规方法 难以得到的 1,8-二苯乙炔基菜的合成.

\section{1 结果与讨论}

\subsection{1,7-二芳基-1,6-二炔的合成}

Sonogashira 偶联反应是形成新的 $\mathrm{C}-\mathrm{C}$ 键及炔基化

\footnotetext{
*E-mail:wym1503@163.com

Received August 20, 2014; revised October 16, 2014; published online November 13, 2014.

Project supported by the National Natural Science Foundation of China (No. 21172076).

国家自然科学基金(No. 21172076)资助项目.
} 
的最有效方法之一, 在合成上有着极为广泛的用途. 在 研究末端二炔的 Sonogashira 反应时, 我们发现, 以 $\mathrm{PdCl}_{2} / \mathrm{CuI}$ 为催化剂, $\mathrm{NEt}_{3}$ 为碱, 在 $\mathrm{THF}$ 溶剂中, 室温摚 拌反应 $6 \mathrm{~h}$ 可以实现芳基碘与末端二炔的偶联, 同时形 成两个 $\mathrm{C}(\mathrm{sp})-\mathrm{C}\left(\mathrm{sp}^{2}\right)$ 键, 二炔上连接的取代基的性质对 反应的收率没有太大的影响. 当二炔上连有吸电子基团 时, 反应也能很好地进行并有着较高的收率, 同样连有 各种取代基的碘代芳烃都能很好地反应, 得到不错的收 率.

碘代芳烃与末端二炔最适宜的反应条件可以有效 地获得 1,6-庚二炔, 为了进一步扩大反应的底物范围, 验证该反应条件的普适性, 我们以溴代芳烃与末端二炔 在上述条件下进行偶联反应, 只要反应温度提高到 $80{ }^{\circ} \mathrm{C}$, 反应时间延长到 $13 \mathrm{~h}$, 也能获得非常满意的结 果(表 1).

表 1 二炔与卤代芳烃 Sonogashira 反应的结果

Table 1 Results of Sonogashira coupling reaction between terminal diacetylenes and aryl halides
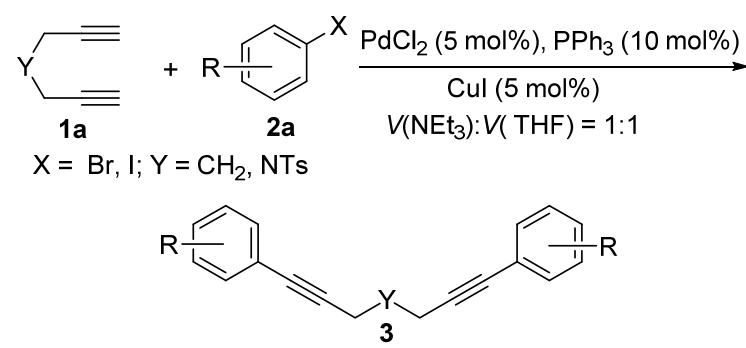

\begin{tabular}{ccclcc}
\hline Entry & $\mathrm{X}$ & $\mathrm{Y}$ & \multicolumn{1}{c}{$\mathrm{R}$} & Product & Yield/\% \\
\hline 1 & $\mathrm{I}$ & $\mathrm{CH}_{2}$ & $\mathrm{H}$ & $\mathbf{3 a}$ & 90 \\
2 & $\mathrm{I}$ & $\mathrm{CH}_{2}$ & $o-\mathrm{F}$ & $\mathbf{3 b}$ & 78 \\
3 & $\mathrm{I}$ & $\mathrm{CH}_{2}$ & $o-\mathrm{Me}$ & $\mathbf{3 c}$ & 83 \\
4 & $\mathrm{I}$ & $\mathrm{CH}_{2}$ & $m-\mathrm{Me}$ & $\mathbf{3 d}$ & 80 \\
5 & $\mathrm{I}$ & $\mathrm{CH}_{2}$ & $p-\mathrm{Br}$ & $\mathbf{3 e}$ & 76 \\
6 & $\mathrm{I}$ & $\mathrm{NTs}^{2}$ & $\mathrm{H}$ & $\mathbf{3 f}$ & 88 \\
$7^{c}$ & $\mathrm{Br}$ & $\mathrm{CH}_{2}$ & $p-\mathrm{F}$ & $\mathbf{3 g}$ & 85 \\
$8^{c}$ & $\mathrm{Br}$ & $\mathrm{CH}_{2}$ & $p-\mathrm{Cl}$ & $\mathbf{3 h}$ & 83 \\
$9^{c}$ & $\mathrm{Br}$ & $\mathrm{CH}_{2}$ & $m-\mathrm{OCH}_{3}$ & $\mathbf{3 i}$ & 74 \\
\hline
\end{tabular}

${ }^{a}$ Reaction conditions: 1a $(0.6 \mathrm{mmol}), \mathbf{2 a}(1.0 \mathrm{mmol}), \mathrm{PdCl}_{2}(5 \mathrm{~mol} \%), \mathrm{PPh}_{3}$ $(10 \mathrm{~mol} \%)$ and $\mathrm{CuI}(5 \mathrm{~mol} \%)$ in $\mathrm{Et}_{3} \mathrm{~N} / \mathrm{THF}(V: V=1: 1)$ at room temperature for $6 \mathrm{~h} .{ }^{b}$ Isolated yields; ${ }^{c} 80{ }^{\circ} \mathrm{C}$ for $13 \mathrm{~h}$.

\section{$1.21,8$-二苯乙炔基萗的合成}

为了进一步验证该 Sonogashira 偶联反应体系的可 行性, 我们以具有较大空间位阻的 1,8 -二碘萗与末端炔 烃在上述条件下进行偶联反应, 也获得了较为满意的结 果(Eq. 1).

\section{2 结论}

以 $\mathrm{PdCl}_{2} / \mathrm{CuI}$ 为催化剂, $\mathrm{NEt}_{3}$ 为碱, 在 $\mathrm{THF}$ 溶剂中, 可以实现芳基卤代物与芳基末端二炔 Sonogashira 偶联

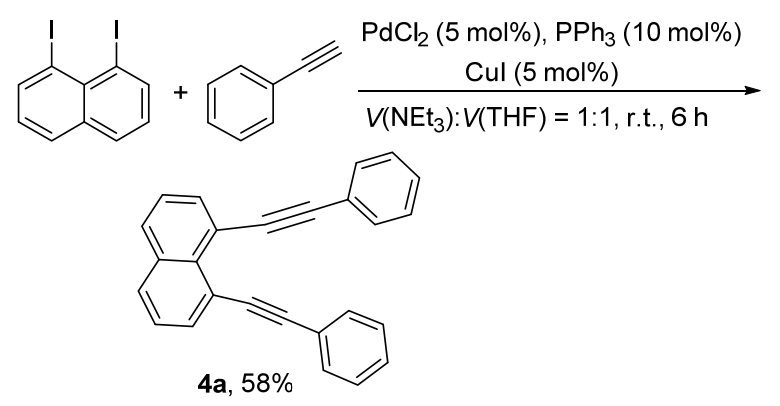

反应，该方法也适用于常规方法难以得到的 1,8-二苯乙 炔基萘的合成. 在 $\mathrm{PdCl}_{2} / \mathrm{CuI} / \mathrm{PPh}_{3} / \mathrm{NEt}_{3}$ 催化体系, THF 溶剂中实现的 Sonogashira 偶联反应, 不仅反应条件温 和, 氮气保护处理简单, 末端炔发生 Glaser 偶联产物少.

\section{3 实验部分}

\section{1 仪器与试剂}

德国 Bruker 公司 $400 \mathrm{MHz}$ DRX-400 核磁共振仪, 内标为 TMS; Analect RFX-65A 型红外光谱仪, 液膜法 或 $\mathrm{KBr}$ 压片法; 美国 Thermo 公司 MAT 95XP 型高分辨 质谱仪; 美国 Frinnigan 公司 Trace DSQ GC-MS 气质联 用仪, 色谱柱: 高弹性石英毛细柱 HP-5, $0.2 \mathrm{~mm} \times 0.25$ $\mu \mathrm{m} \times 30 \mathrm{~mm}, \mathrm{EI}$ 源 (电子能量: $70 \mathrm{ev}$ ). 所用的化学原料 试剂全部从 Alfa, Aldrich 等公司购买, 没有进一步的纯 化; 常用溶剂购自广州试剂公司; 薄层层析硅胶由硅胶 GF254(青岛海洋化工厂)和 CMC 自制; 柱层析用硅胶为 化学纯试剂(青岛海洋化工厂).

\section{2 实验方法}

\subsubsection{4-甲基- $N, N$-二(2-炔丙基)苯磺酰胺的合成}

往试管中相继加入 $\mathrm{K}_{2} \mathrm{CO}_{3}(276 \mathrm{mg}, 2$ equiv)、对甲 基苯磺酰胺 $(171 \mathrm{mg}, 1 \mathrm{~mol}) 、 \mathrm{DMF}(2 \sim 5 \mathrm{~mL})$ 和炔丙澳 (354 mg, $3 \mathrm{~mol}$ ), 室温搅拌过夜. 用 TLC 检测反应完成 后, 加入石油醚, 过滤, 减压除去溶剂. 薄层色谱分离 可得二炔产物, 产物的分子量经 MS 确认.

\subsubsection{1,7-二芳基-1,6-二炔的合成}

分别将 $\mathrm{PdCl}_{2}$ (5 mol \%), $\mathrm{CuI}(5 \mathrm{~mol} \%), \mathrm{PPh}_{3}(10$ $\mathrm{mol} \%$ )依次加入试管中, 再加入 $1 \mathrm{~mL}$ 三乙胺, $1 \mathrm{~mL}$ 四氢 呋喃, 后继加入卤化物 $(1.0 \mathrm{mmol})$ 、末端㶧 $(0.6 \mathrm{mmol})$, 并接好充着一大气压氮气的气球, 底物为磑化物时室温 搅拌 $6 \mathrm{~h}$; 而溴化物与末端炔的偶联需要 $80{ }^{\circ} \mathrm{C}$ 摚拌 13 h. 用 TLC 检测反应完成后, 往反应混合液中加入 10 $\mathrm{mL}$ 饱和食盐水, 再用乙酸乙酯 $(10 \mathrm{~mL} \times 3)$ 进行萃取, 合并有机层并用饱和食盐水进行洗涤, 用无水 $\mathrm{MgSO}_{4}$ 进行干燥. 所得到的有机相减压蒸馏除去大部分溶剂, 粗产物用柱色谱进行分离提纯. 产物的结构经 ${ }^{1} \mathrm{H} N \mathrm{NMR}$, ${ }^{13} \mathrm{C}$ NMR, GC-MS 和 IR 进行分析确认. 
1,7-二苯基-1,6-庚二炔(3a): $110 \mathrm{mg}$ 黄色粘稠, 收率 $90 \% .{ }^{1} \mathrm{H}$ NMR $\left(\mathrm{CDCl}_{3}, 400 \mathrm{MHz}\right) \delta: 7.39 \sim 7.25(\mathrm{~m}, 10 \mathrm{H})$, $2.58(\mathrm{t}, J=8.0 \mathrm{~Hz}, 4 \mathrm{H}), 1.89(\mathrm{t}, J=8.0 \mathrm{~Hz}, 2 \mathrm{H}) ;{ }^{13} \mathrm{C} \mathrm{NMR}$ $\left(\mathrm{CDCl}_{3}, 100 \mathrm{MHz}\right) \delta: 131.66,128.29,127.71,123.92$, 89.28, 81.35, 28.05, 18.76; IR (KBr) v: 2930, 1502, 1116, 973, $826 \mathrm{~cm}^{-1}$; MS (EI, $70 \mathrm{eV)} \mathrm{m} / \mathrm{z}: 244,228,215,167$, $115 ;{ }^{1} \mathrm{H}$ NMR 和 ${ }^{13} \mathrm{C}$ NMR 与 Liu 等的数据 ${ }^{[17]}$ 一致.

1,7-二(2-氟苯基)-1,6-庚二炔(3b): $109 \mathrm{mg}$ 黄色粘稠, 收率 78\%. ${ }^{1} \mathrm{H}$ NMR $\left(400 \mathrm{MHz}, \mathrm{CDCl}_{3}\right) \delta: 7.42 \sim 7.37$ (m, $2 \mathrm{H}), 7.26 \sim 7.20(\mathrm{~m}, 2 \mathrm{H}), 7.06 \sim 7.01(\mathrm{~m}, 4 \mathrm{H}), 2.65(\mathrm{t}, J=$ $8.0 \mathrm{~Hz}, 4 \mathrm{H}), 1.98 \sim 1.90(\mathrm{~m}, 2 \mathrm{H}) ;{ }^{13} \mathrm{C}$ NMR $(101 \mathrm{MHz}$, $\left.\mathrm{CDCl}_{3}\right) \delta: 162.87(\mathrm{~d}, 249 \mathrm{~Hz}), 133.59,133.57,129.35$, $129.27,123.86,123.82,115.48,115.27,112.38,112.22$, 94.66, 94.62, 74.67, 27.70, 18.83; IR (KBr) v: 2930, 1502, $1156,973,836 \mathrm{~cm}^{-1}$; MS (EI, $70 \mathrm{eV}$ ) $\mathrm{m} / \mathrm{z}: 280,264,251$, 244, 207, 183; HRMS (EI) calcd for $\mathrm{C}_{19} \mathrm{H}_{14} \mathrm{~F}_{2} 280.1064$, found 280.1060 .

1,7-二(2-甲基苯基)-1,6-庚二炔(3c)：126 mg 黄色粘 稠, 收率 $83 \% .{ }^{1} \mathrm{H}$ NMR $\left(400 \mathrm{MHz}, \mathrm{CDCl}_{3}\right) \delta: 7.37(\mathrm{~d}, J=$ $8.0 \mathrm{~Hz}, 2 \mathrm{H}), 7.16 \sim 7.07(\mathrm{~m}, 6 \mathrm{H}), 2.65(\mathrm{t}, J=8.0 \mathrm{~Hz}, 4 \mathrm{H})$, $2.42(\mathrm{~s}, 6 \mathrm{H}), 1.96 \sim 1.89(\mathrm{~m}, 2 \mathrm{H}) ;{ }^{13} \mathrm{C}$ NMR $(101 \mathrm{MHz}$, $\left.\mathrm{CDCl}_{3}\right) \delta: 140.01,131.90,129.37,127.68,125.50,123.67$, 93.17, 80.22, 27.95, 21.07, 18.73; IR (KBr) v: 2930, 1502, $1111,945,820 \mathrm{~cm}^{-1}$; MS (EI, $70 \mathrm{eV}$ ) $\mathrm{m} / \mathrm{z}: 272,257,229$, $215,165,141,128,115$; HRMS (EI) calcd for $\mathrm{C}_{21} \mathrm{H}_{20}$ 272.1565 , found 272.1573 .

1,7-二(3-甲基苯基)-1,6-庚二炔(3d): $108 \mathrm{mg}$ 黄色粘 稠, 收率 $80 \% .{ }^{1} \mathrm{H} \mathrm{NMR}\left(\mathrm{CDCl}_{3}, 400 \mathrm{MHz}\right) \delta: 7.23 \sim 7.14$ $(\mathrm{m}, 6 \mathrm{H}), 7.07(\mathrm{~d}, J=8.0 \mathrm{~Hz}, 2 \mathrm{H}), 2.58(\mathrm{t}, J=8.0 \mathrm{~Hz}, 4 \mathrm{H})$, $2.30(\mathrm{~s}, 6 \mathrm{H}), 1.93 \sim 1.86(\mathrm{~m}, 2 \mathrm{H}) ;{ }^{13} \mathrm{C}$ NMR $\left(\mathrm{CDCl}_{3}, 101\right.$ MHz) $\delta: 137.87,132.24,128.65,128.54,128.14,123.67$, $88.85,81.39,28.07,21.22,18.72$; IR (KBr) v: 2930, 1502, $1124,943,826 \mathrm{~cm}^{-1}$; MS (EI, $70 \mathrm{eV}$ ) $\mathrm{m} / z: 272,257,242$, $165,128,115,{ }^{1} \mathrm{H}$ NMR 和 ${ }^{13} \mathrm{C}$ NMR 与 Jiang 等的数据 ${ }^{[16 c]}$ 一致.

1,7-二(4-溴苯基)-1,6-庚二炔(3e): $153 \mathrm{mg}$ 黄色粘稠, 收率 76\%. ${ }^{1} \mathrm{H}$ NMR $\left(\mathrm{CDCl}_{3}, 400 \mathrm{MHz}\right) \delta: 7.41(\mathrm{~d}, J=8.0$ $\mathrm{Hz}, 4 \mathrm{H}), 7.25$ (d, $J=8.0 \mathrm{~Hz}, 4 \mathrm{H}), 2.57(\mathrm{t}, J=8.0 \mathrm{~Hz}, 4 \mathrm{H})$, $1.93 \sim 1.86(\mathrm{~m}, 2 \mathrm{H}) ;{ }^{13} \mathrm{C}$ NMR $\left(\mathrm{CDCl}_{3}, 100 \mathrm{MHz}\right) \delta$ : 133.06, 131.46, 122.76, 121.80, 90.36, 80.35, 27.68, 18.72; IR (KBr) $v: 2930,1502,1116,974,820 \mathrm{~cm}^{-1}$; MS (EI, 70 eV) $m / z$ : 402, 241, 239, 165, 115; HRMS (EI) calcd for $\mathrm{C}_{19} \mathrm{H}_{14} \mathrm{Br}_{2} 401.9442$, found 401.9448 .

4-甲基- $N, N$-二(3-苯基-2-炔丙基)苯甲酰胺(3f)：176 $\mathrm{mg}$ 黄色固体, 收率 $88 \%$. m.p. $113.5 \sim 114.6{ }^{\circ} \mathrm{C} ;{ }^{1} \mathrm{H}$ NMR $\left(400 \mathrm{MHz}, \mathrm{CDCl}_{3}\right) \delta: 7.83(\mathrm{~d}, J=8.0 \mathrm{~Hz}, 2 \mathrm{H}), 7.33 \sim 7.24$ $(\mathrm{m}, 12 \mathrm{H}), 4.48$ (s, 4H), 2.35 (s, 3H); ${ }^{13} \mathrm{C}$ NMR $(100 \mathrm{MHz}$, $\left.\mathrm{CDCl}_{3}\right) \delta: 143.85,135.45,131.70,129.61,128.53,128.19$, $128.01,122.25,85.82,81.70,37.54,21.42$; IR (KBr) $v$ : 1349, 1168, 895, $756 \mathrm{~cm}^{-1}$; MS (EI, $\left.70 \mathrm{eV}\right) \mathrm{m} / z$ : 399, 182, $91 ;{ }^{1} \mathrm{H}$ NMR 和 ${ }^{13} \mathrm{C} \mathrm{NMR}$ 与 Liu 等的数据 ${ }^{[17]}$ 一致.

1,7-二(4-氟苯基)-1,6-庚二炔 $(\mathbf{3 g}$ ): $119 \mathrm{mg}$ 黄色粘稠, 收率 85\%. ${ }^{1} \mathrm{H}$ NMR $\left(400 \mathrm{MHz}, \mathrm{CDCl}_{3}\right) \delta: 7.41 \sim 7.37(\mathrm{~m}$, $4 \mathrm{H}), 6.99(\mathrm{t}, J=8.4 \mathrm{~Hz}, 4 \mathrm{H}), 2.59(\mathrm{t}, J=6.8 \mathrm{~Hz}, 4 \mathrm{H})$, $1.95 \sim 1.88(\mathrm{~m}, 2 \mathrm{H}) ;{ }^{13} \mathrm{C}$ NMR $\left(100 \mathrm{MHz}, \mathrm{CDCl}_{3}\right) \delta$ : 162.15 (d, $25 \mathrm{~Hz}), 133.37$ (d, $8 \mathrm{~Hz}), 119.89$ (d, $4 \mathrm{~Hz})$, 115.43 (d, $22 \mathrm{~Hz}), 88.77,80.25,27.89$, 18.62; IR (KBr) v: 2934, 1502, 1123, 980, $836 \mathrm{~cm}^{-1}$; MS (EI, $\left.70 \mathrm{eV}\right) \mathrm{m} / z$ : $280,244,183,133,83 ;{ }^{1} \mathrm{H}$ NMR 和 ${ }^{13} \mathrm{C}$ NMR 与 Jiang 等的

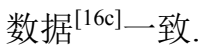

1,7-二(4-氯苯基)-1,6-庚二炔 $(\mathbf{3 h}$ ): $129 \mathrm{mg}$ 黄色粘稠, 收率 $83 \% .{ }^{1} \mathrm{H}$ NMR $\left(400 \mathrm{MHz}, \mathrm{CDCl}_{3}\right) \delta: 7.26(\mathrm{~d}, J=8.0$ $\mathrm{Hz}, 4 \mathrm{H}), 7.19$ (d, $J=8.0 \mathrm{~Hz}, 4 \mathrm{H}), 2.52(\mathrm{t}, J=7.2 \mathrm{~Hz}, 4 \mathrm{H})$, $1.87 \sim 1.80(\mathrm{~m}, 2 \mathrm{H}) ;{ }^{13} \mathrm{C}$ NMR $\left(100 \mathrm{MHz}, \mathrm{CDCl}_{3}\right) \delta$ : 133.64, 132.82, 128.54, 122.32, 90.16, 80.31, 27.76, 18.70; IR (KBr) v: 2934, 1502, 1123, 980, $836 \mathrm{~cm}^{-1}$; MS (EI, 70 eV) $m / z: 312,277,242,199,145,116 ;{ }^{1} \mathrm{H}$ NMR 和 ${ }^{13} \mathrm{C}$ NMR与Jiang等的数据 ${ }^{[16 c]}$ 一致.

1,7-二(3-甲氧基苯基)-1,6-庚二炔(3i)：106 mg 黄色 粘稠, 收率 74\%. ${ }^{1} \mathrm{H}$ NMR $\left(400 \mathrm{MHz}, \mathrm{CDCl}_{3}\right) \delta: 7.21(\mathrm{t}$, $J=8.0 \mathrm{~Hz}, 2 \mathrm{H}), 7.04 \sim 6.96(\mathrm{~m}, 4 \mathrm{H}), 6.86(\mathrm{~d}, J=8.4 \mathrm{~Hz}$, $2 \mathrm{H}), 3.80(\mathrm{~s}, 6 \mathrm{H}), 2.61(\mathrm{t}, J=7.2 \mathrm{~Hz}, 4 \mathrm{H}), 1.97 \sim 1.90(\mathrm{~m}$, $2 \mathrm{H}) ;{ }^{13} \mathrm{C}$ NMR $\left(100 \mathrm{MHz}, \mathrm{CDCl}_{3}\right) \delta: 159.33,129.27$, 124.86, 124.16, 116.47, 114.29, 89.08, 81.21, 55.24, 27.95, 27.50, 18.71; IR (KBr) v: 2930, 1502, 1120, 945, 828 $\mathrm{cm}^{-1}$; MS (EI, $70 \mathrm{eV}$ ) m/z: 304, 261, 202, 165, 115, 76, HRMS (EI) calcd for $\mathrm{C}_{21} \mathrm{H}_{20} \mathrm{O} 304.1463$, found 304.1471.

\section{$3.2 .31,8$-二碘菜的合成}

往圆底烧瓶中加入 1,8 -二菜胺 $(995 \mathrm{mg}, 6.3 \mathrm{mmol})$, $6.9 \mathrm{~mol} / \mathrm{L}$ 的硫酸 $10 \mathrm{~mL}$, 将烧瓶置于低温反应槽中降温 至 $-20{ }^{\circ} \mathrm{C}$, 将配制好的 $\mathrm{NaNO}_{2}$ 溶液 $\left(1.08 \mathrm{~g} \mathrm{NaNO}_{2}\right.$, 加 水 $4 \mathrm{~mL}$ 溶解)、 $\mathrm{KI}$ 溶液(5.38 g KI, 加水 $4.5 \mathrm{~mL}$ 溶解)加 入反应烧瓶中, 然后缓慢加入浓硫酸, 搅拌 $20 \mathrm{~min}$, 取 出圆底烧瓶于油浴锅迅速加热至 $80{ }^{\circ} \mathrm{C}$, 冷却至室温, 加入氢氧化钠中和反应混合液呈中性, 过滤, 收集黑色 固体物质, 研磨, 用煮沸的乙醚萃取, 醚层分别加入 $10 \%$ 盐酸、饱和硫代亚硫酸钠溶液、氢氧化钠溶液进行 萃取，收集乙醚层并用饱和食盐水进行洗涤，用无水 $\mathrm{MgSO}_{4}$ 进行干燥, 得到的有机相减压蒸馏除去大部分 溶剂, 粗产物用柱色谱进行分离提纯, 产物的分子量经 
MS 确认.

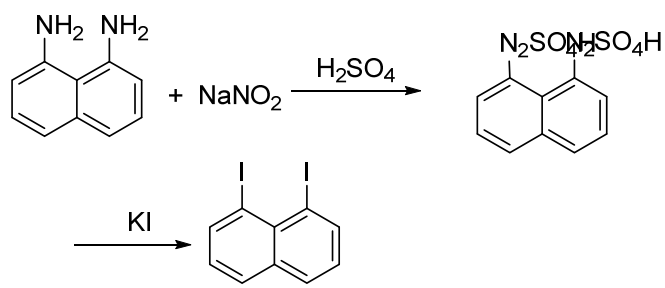

图式 1 1,8-二碘荎的合成反应式

Scheme 1 Synthesis reaction for 1,8-diiodonaphthalene

\section{2 .4 1,8-二苯乙炔基䓠的合成}

分别将 $\mathrm{PdCl}_{2}$ (5 mol\%), CuI (5 mol\%), $\mathrm{PPh}_{3}(10$ $\mathrm{mol} \%$ )依次加入试管中, 再加入 $1 \mathrm{~mL}$ 三乙胺, $1 \mathrm{~mL}$ 四氢 呋喃, 后继加入 1,8 -二碘菜 $(1.0 \mathrm{mmol})$ 、苯乙炔 $(2.4$ $\mathrm{mmol})$, 室温搅拌 $6 \mathrm{~h}$. 用 TLC 检测反应完成后, 往反应 混合液中加入 $10 \mathrm{~mL}$ 饱和食盐水, 再用乙酸乙酯萃取 (10 mL X3), 合并有机层并用饱和食盐水进行洗涤, 用 无水 MgSO4 进行干燥. 所得到的有机相减压蒸馏除去 大部分溶剂, 粗产物用柱色谱进行分离提纯. 1,8-二苯 乙炔基菜(4a): 浅黄色固体, 收率 58\%. m.p. 105.6 $106.4{ }^{\circ} \mathrm{C} ;{ }^{1} \mathrm{H}$ NMR $\left(400 \mathrm{MHz}, \mathrm{CDCl}_{3}\right) \delta: 7.83$ (dd, $J=$ 20.4, 7.7 Hz, 4H), 7.44 (t, $J=8.0 \mathrm{~Hz}, 2 \mathrm{H}), 7.35$ (d, $J=8.0$ $\mathrm{Hz}, 4 \mathrm{H}), 7.23 \sim 7.17(\mathrm{~m}, 2 \mathrm{H}), 7.10(\mathrm{~d}, J=8.0 \mathrm{~Hz}, 4 \mathrm{H}) ;{ }^{13} \mathrm{C}$ NMR (101 MHz, $\left.\mathrm{CDCl}_{3}\right) \delta: 134.96,134.16,131.62$, 131.47 , 129.66, 127.96, 127.90, 125.62, 123.79, 120.89, 96.70, 89.79; IR (KBr) v: 3037, 1602, 1484, 781, 690, 441 $\mathrm{cm}^{-1}$; MS (EI, $\left.70 \mathrm{eV}\right) \mathrm{m} / z: 328,163,150 ;{ }^{1} \mathrm{H}$ NMR 和 ${ }^{13} \mathrm{C}$ $\mathrm{NMR}$ 与 Wang 等的数据 ${ }^{[18]}$ 一致.

\section{References}

[1] (a) Chinchilla, R.; Nájera, C. Chem. Rev. 2007, 107(3), 874. (b) Chinchilla, R.; Nájera, C. Chem. Soc. Rev. 2011, 40, 5084.

[2] (a) He, Y.; Cai, C. J. Org. Chem. 2011, 696(13), 2689. (b) Suzuka, T.; Okada, Y.; Ooshiro, K.; Uozumi, Y. Tetrahedron 2010, 66(5), 1064

(c) Wang, Y.; Liu, J. H.; Xia, C. G. Tetrahedron Lett. 2011, 52(14), 1587. (d) Bakherad, M.; Keivanloo, A.; Kalantar, Z.; Jajarmi, S. Tetrahedron Lett. 2011, 52(2), 228.

(e) Nazario, C. E. D.; Santana, A. S.; Kawasoko, C. Y.; Carollo, C. A.; Hurtado, G. R.; Viana, L. H.; Barbosa, S. L.; Guerrero, P. G.; Marques, F. A.; Dabdoub, V. B.; Dabdoub, M. J.; Baroni, A. C. M. Tetrahedron Lett. 2011, 52(32), 4177.

[3] Kanuru, V. K.; Humphrey, S. M.; Kyffin, J. M. W.; Jefferson, D. A.; Burton, J. W.; Armbrüster, M.; Lambert, R. M. Dalton Trans. 2009, 37, 7602.

[4] Wang, L.; Li, P.-H.; Zhang, Y.-C. Chem. Commun. 2004, 5, 514.

[5] Vechorkin, O.; Barmaz, D.; Proust, V.; Hu, X.-L. J. Am. Chem. Soc. 2009, $131(34), 12078$.

[6] (a) Huang, H.; Jiang, H.-L.; Chen, K.-X.; Liu, H. J. Org. Chem. 2008, 73(22), 9061.

(b) Carril, M.; Correa, A.; Bolm, C. Angew. Chem., Int. Ed. 2008, 47(26), 4862.

(c) Xie, X.; Xu, X.; Li, H.; Xu, X.; Yang, J.; Lia, Y. Adv. Synth. Catal. 2009, 351, 1263.

(d) Liu, Y.; Yang, J.; Bao, W. Eur. J. Org. Chem. 2009, 5317.

[7] Trost, B. M.; Rudd, M. T. A. J. Am. Chem. Soc., 2003, 125(38), 11516.

[8] Tanaka, K.; Otake, Y.; Wada, A.; Noguchi, K.; Hirano, M. Org. Lett. 2007, 9(11), 2203.

[9] Tanaka, K.; Otake, Y.; Hirano, M. Org. Lett. 2007, 9(20), 3953.

[10] (a) Yamamoto, Y.; Mori, S.; Shibuya, M. Chem.-Eur. J. 2013, 19(36), 12034.

(b) Robinson, J. M.; Sakai, T.; Okano, K.; Kitawaki, T.; Danheiser, R. L. J. Am. Chem. Soc. 2010, $132(32), 11039$.

[11] Varela, J. A.; Rubín, S. G.; Rodríguez, C. G.; Castedo, L.; Saá, C. J. Am. Chem. Soc. 2006, 128(29), 9262.

[12] Wen, Y.-M.; Zhu, S.-F.; Jiang, H.-F.; Wang, A.-Z.; Chen, Z.-W. Synlett 2011, 7, 1023

[13] Tanaka, K.; Osaka, T.; Noguchi, K.; Hirano, M. Org. Lett. 2007, 9(7), 1307

[14] Zhang, K.-N.; Louie, J. J. Org. Chem. 2011, 76(11), 4686

[15] Yamamoto, Y.; Matsui, K.; Shibuya, M. Org. Lett. 2014, 16(6), 1806.

[16] (a) Novák, P.; Č́́halová, S.; Otmar, M.; Hocek, M.; Kotora, M. Tetrahedron 2008, 64(22), 5200.

(b) Rao, W.; Joo Koh, M.; Li, D.; Hirao, H.; Chan, P. W. H. J. Am. Chem. Soc. 2013, 135(21), 7926.

(c) Zhou, P.; Zhen, M.-F.; Jiang, H.-F.; Li, X.-W.; Qi, C.-R. J. Org. Chem. 2011, 76(11), 4759.

[17] Lian, J.-J.; Chen, P.-C.; Lin, Y.-P.; Ting, H.-C.; Liu, R.-S. J. Am. Chem. Soc. 2006, 128, 11372.

[18] Chen, X.-P.; Lu, P.; Wang, Y.-G. Chem. Eur. J. 2011, 17, 8105. 